\title{
Anticipating Atrial Fibrillation Signal Using Efficient Algorithm
}

https://doi.org/10.3991/ijoe.v17i02.19183

\author{
Mohand Lokman Al-dabag $(\bowtie)$ \\ Northern Technical University, Mosul, Iraq \\ mohandaldabag@ntu.edu.iq \\ Haider Th. Salim ALRikabi \\ Wasit University, Wasit, Iraq \\ Raid Rafi Omar Al-Nima \\ Northern Technical University, Mosul, Iraq
}

\begin{abstract}
One of the common types of arrhythmia is Atrial Fibrillation (AF), it may cause death to patients. Correct diagnosing heart problems through examining the Electrocardiogram (ECG) signal will lead to prescribing the right treatment for a patient. This study proposes a system that distinguishes between the normal and AF ECG signals. First, this work provides a novel algorithm for segmenting the ECG signal for extracting a single heartbeat. The algorithm utilizes low computational cost techniques to segment the ECG signal. Then, useful preprocessing and feature extraction methods are suggested. Two classifiers, Support Vector Machine (SVM) and Multilayer Perceptron (MLP), are separately used to evaluate the two proposed algorithms. The performance of the last proposed method with the two classifiers (SVM and MLP) shows an improvement of about (19\% and $17 \%$, respectively) after using the proposed segmentation method so it became $96.2 \%$ and $97.5 \%$, respectively.
\end{abstract}

Keywords - ECG signal, Support Vector Machine, Multilayer Perceptron, Atrial Fibrillation, Cross-Correlation

\section{Introduction}

Diagnosing various kinds of heart diseases was performed through ECG signals, but these signals are disturbed with several types of noises, and this may yield incorrect interpretations of these signals. Hence, eliminating noises from the ECG signals have been attracted significant attention in order to obtain clean signals to extract undisturbed features from ECG signals with acceptable accuracies for medical purposes.[3, 4]. AF is one of the abnormal cardiac arrhythmia types that affects five million people in the USA. Recent studies expected that 700,000 people in the USA may have undiagnosed AF. Therefore, more efforts and studies are needed to develop accurate non-invasive technology for expecting AF signals accurately. These technologies enable patients to 
track and predict the occurrence of AF signals[5, 6]. Untreated AF can cause a risk of stroke, dizziness, or dyspnea. In the worst case, patients may die. Therefore, predicting $\mathrm{AF}$ is essential for medical purposes [7, 8].

There are several suggested approaches for denoising and extracting features to classify different diseases from ECG signals. Some of them are based on Fourier transform to extract ECG features in a frequency domain[3]. Another work suggested time-frequency approaches with Independent Component Analysis (ICA), which provides features to Neural Network (NN) for classifying ECG signals in some medical fields[9]. Some researchers preferred using Butterworth band-pass filter with a second-order dynamic system. They gave enough features to statistical T-test classifier for classifying ECG signals[10]. Another study claimed using Deep Belief Network (DBN) to classify filtered ECG signals and provided an acceptable classification rate (about 84\%)[11]. Other researchers proposed a method base on filtering the ECG signal, detecting peak algorithm, statistically extracting features, and using four classifiers of SVM, Adaboost, NN, and Naïve Bayes[12]. Another study converted the pre-processed one-dimensional ECG signal into a two-dimensional ECG signal and fed it to Faster Regions with a Convolutional Neural Network (Faster R-CNN)[13]. Another article utilized three different datasets (ECG-ID, MIT-BIH Arrhythmia database and UCI Machine Learning Repository (Gait)) for classification using three classifiers; K-nearest neighbor (KNN) relying on Euclidean distance, PNN (Probabilistic Neural Network), RBF (Radial Basis Function) and Support Vector Machine (SVM), relying on One-against-all (OAA). Two modalities are merged to construct features for both signals ( ECG and Gait) to test 18 subject signals[14]. A normal ECG wave consists of three parts: P wave, QRS wave, and $\mathrm{T}$ wave. The $\mathrm{P}$ wave is caused by atrial contraction, while, the ventricular recovery produces the T wave. The QRS wave is composed of three intervals: Q, R, and S intervals. The $\mathrm{Q}$ interval is the downward deflection before the ventricular contraction, while, $\mathrm{R}$ interval is the peak value of the ventricular contraction, and the $\mathrm{S}$ interval is the downward deflection after the ventricular contraction. See Figure $1[3,14,15]$. This study proposed an algorithm to detect R points of ECG signal and use it as a reference point for extracting a set of a single heartbeat. Then, a suggestion of the Preprocessing and feature extraction algorithm is also made to classify two ECG signal classes (normal and AF) using two classification algorithms (SVM and MLP). The results show the proposed ECG segmentation algorithm has a valuable impact on the performance of the classification procedure. This article is composed of several sections: section 2 provides a description of the two proposed methods and it also gives a theoretical introduction about materials and methods utilized in this paper, section 3 discusses the results and the last section presents the conclusion. 


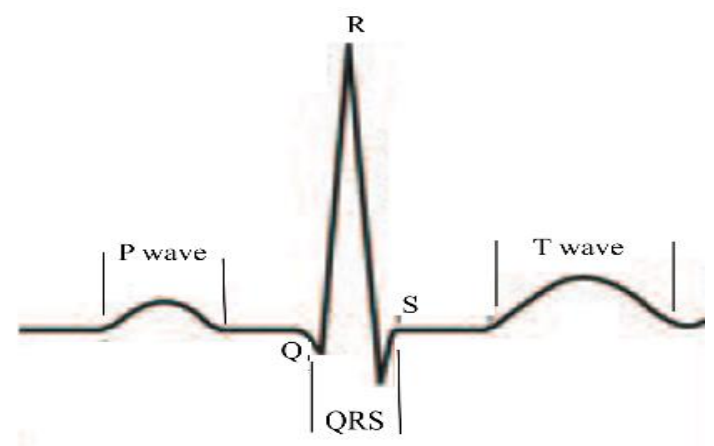

Fig. 1. An ECG signal with its typical intervals [7].

\section{Materials and Methods}

\subsection{The suggested algorithm}

This algorithm represents the preprocessing and feature extraction algorithm which is involved in five stages; denoising based on a wavelet, normalization, cross-correlation, feature extraction, and classification using MLP or SVM. Figure 2 illustrates the suggested preprocessing and feature extraction procedure.

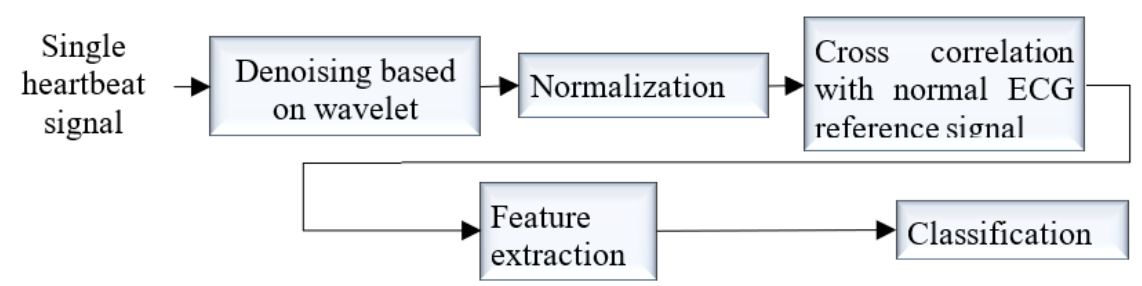

Fig. 2. block diagram of the ECG classification procedure

Firstly, performing the ECG denoising based on the wavelet transform. The ECG signal was decomposed by 7 levels using the Daubechies 2 (Db2) filter. Then, the ECG signal was reconstructed after excluding the coefficients of level 7, see Figure 3. 


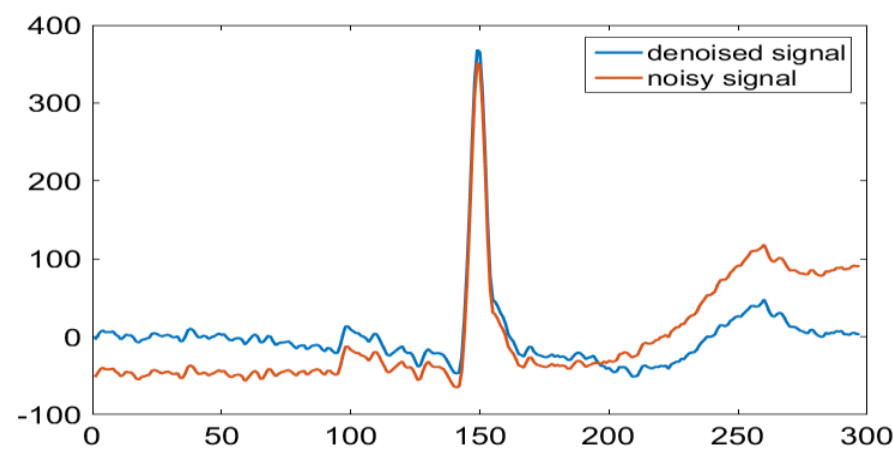

No. of samples in each signal

Fig. 3. De noised ECG signal and noisy signal

Secondly, normalization is employed where the signal is divided by its absolute maximum value. Thirdly, the cross-correlation is applied by correlating the ECG signal with a reference of normal ECG signal. Fourthly, ten feature extraction methods of min, max, mean, median, mode, 1st quartile, 2nd quartile, Standard Deviation (SD), range, and entropy are utilized. Finally, classifying the two ECG classes by using one of the two classifiers MLP or SVM.

\subsection{Proposed ECG segmentation algorithm}

The segmentation of ECG signals to a single heartbeat involves determining the correct heartbeat interval and its peaks. Since R point is the peak value of the ECG signal so the traditional segmentation algorithms and this algorithm use it to determine each ECG heartbeat boundaries. In the proposed algorithm, the thresholding technique is used to determine the R point and QRS interval of the ECG signal. The threshold estimation is given by the following equation.

$$
\tilde{s}(t)= \begin{cases}s(t) & s(t)>\sigma \\ 0 & s(t)<\sigma\end{cases}
$$




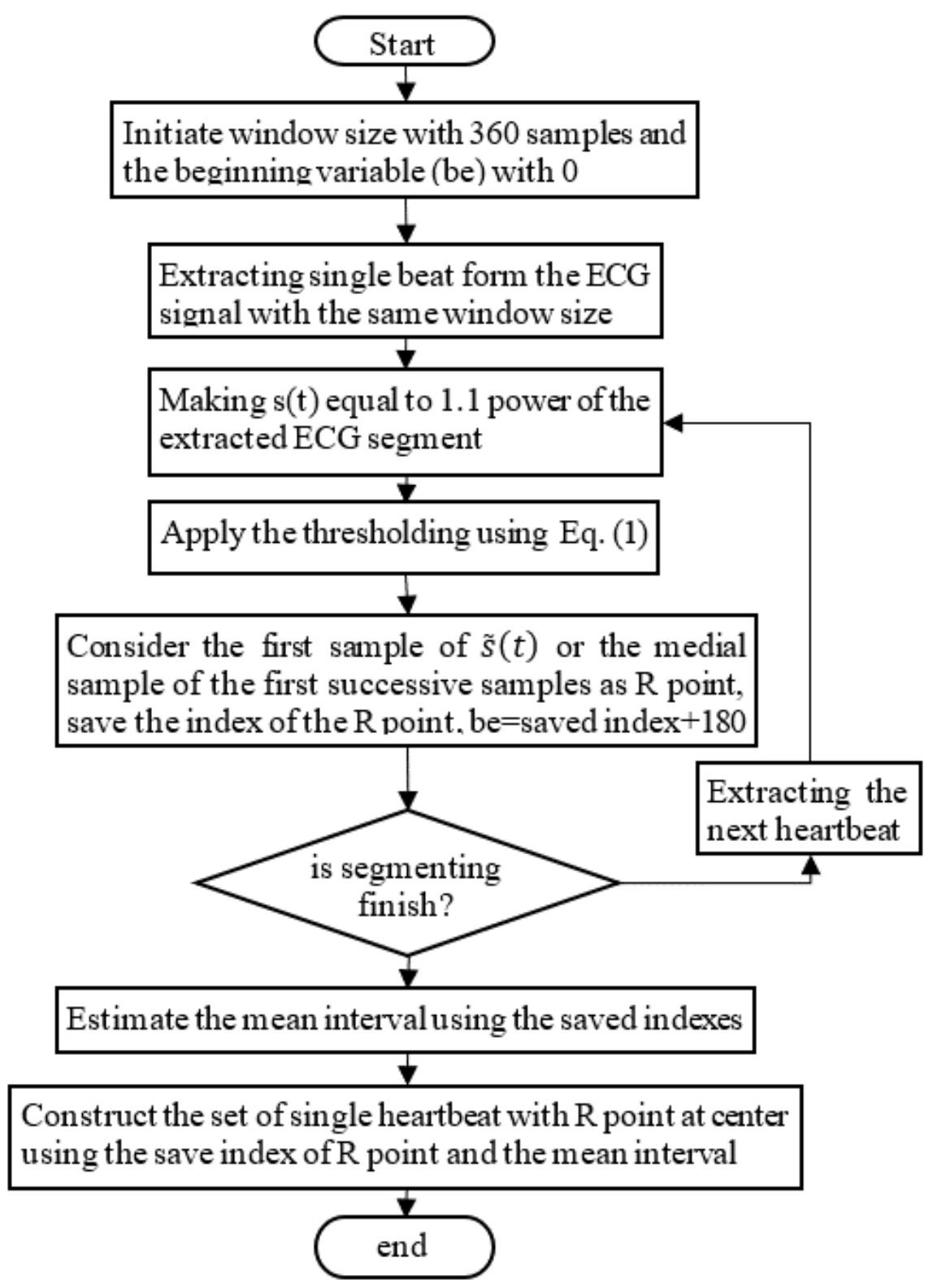

Fig. 4. Flowchart of the proposed ECG segmentation algorithm

where $\mathrm{s}(\mathrm{t})$ is the ECG signal raised to the base of 1.1 and $\sigma$ is the mean plus the standard deviation (SD) of $\mathrm{s}(\mathrm{t})$. The following steps illustrate the algorithm 
1. Initiate the size of the segmentation window to 360 samples $(1 \mathrm{sec}$.) with variable (be $=0$ ).

2. Construct the current ECG segment from the ECG signal.

3. Make each segment exponent of (1.1).

4. Apply Eq. (1) to find samples greater than the threshold.

5. Consider the medial sample of the first successive points as the R point and save its index.

6. Make (be) variable equal to the index of the considered R point plus 180 (360/2).

7. Check if the ECG segmentation is finished. If not go to step 2 otherwise continue.

8. Estimate the periods between two successive R points to find the mean interval of the whole ECG dataset.

9. Use the extracted information (the index of $\mathrm{R}$ points and the mean interval) for segmenting the original ECG signal and make the R points lie at the center of each ECG segment, see Figure 4.

\subsection{Support vector machine}

The SVM is a machine learning classifier. It has been exploited in different fields as in [16]. The target of the SVM is to determine a hyperplane in $\mathrm{N}$-dimensional space ( $\mathrm{N}$ is the number of features). This hyperplane distinguishes between the data of classes. For example, to separate data of two classes, there are infinite hyperplanes that could be chosen for the separation, but there is only one hyperplane that has maximum margins between the classes, as shown in Figure 5 [17-19]. In this paper, The Radial base kernel function with an automatic Kernel Scale (KernelScale) is used for the SVM.

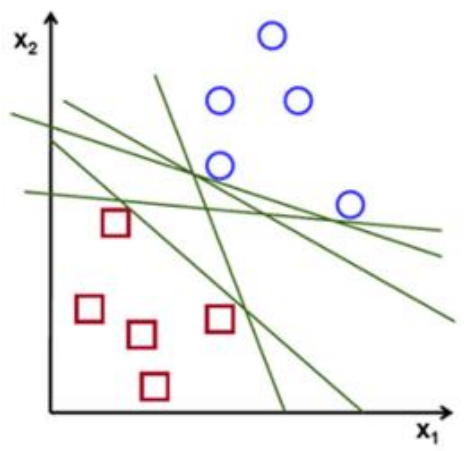

(a) Before the classification

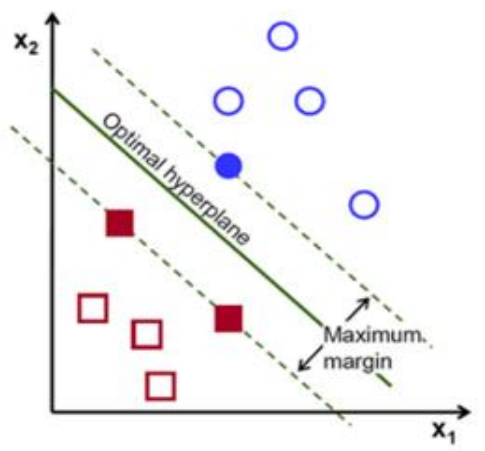

(b) After the classification [11].

Fig. 5. Classifying two classes using the SVM 


\subsection{Multilayer neural network}

The MLP is a type of multilayer NN. Typically, these networks are structured from at least two computational layers and the input layer. The computational layers are the output layer and at least one hidden layer. Figure 6 shows a MLP network[20, 21]. The learning algorithm updates the connection weights of the computation layers. There is a relatively simple learning algorithm for a predefined neural network. This algorithm is known as backpropagation. This is the most popular learning algorithm among hundreds of different learning algorithms. The algorithm has two phases. First, the training set is supplied to the input layer of MLP, and its multilayers forward the training set from layer to layer until reaching the output layer to generate the output pattern. In the second phase, a comparison is made between the output pattern with the desired output to calculate the output error. The error (if exists) is then backward from the output layer to the input layer. While the error is backward, the weights of the network are updated. This procedure is repeated until the error is minimized to a specific range[20][23,22].

This study used the MLP classifier with one hidden layer using the Levenberg-Marquardt backpropagation training algorithm with an error rate set to 0.001 and the evaluation function is Mean Square Error (MSE).

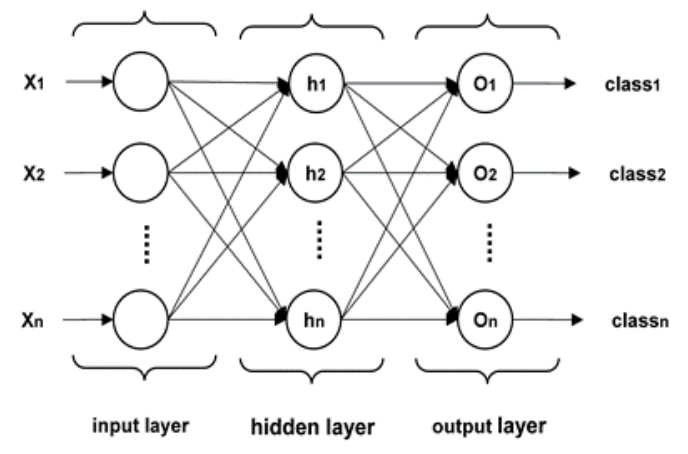

Fig. 6. MLP internal structure with two hidden layers[2]

\subsection{Discrete Wavelet Transform (DWT)}

DWT is widely used in biomedical engineering due to its property of revealing timefrequency components of the signal. It has various types of wavelet filters to decompose a given signal into detail and approximation coefficients[24]. The signal is processed by a low pass filter and then down-sampled by two to produce the approximation coefficients of the next level. The detail coefficients are produced from filtering the signal by a high pass filter and downsampled the output by two. The detail components are kept and the approximation can farther be de-composed to the next level by passing it through the same low pass and high pass filters then downsampled the out coming by 2. This process can be repeated to decompose the signal to the target scale. Figure 7 shows two levels of DWT decomposition[25, 26]. 


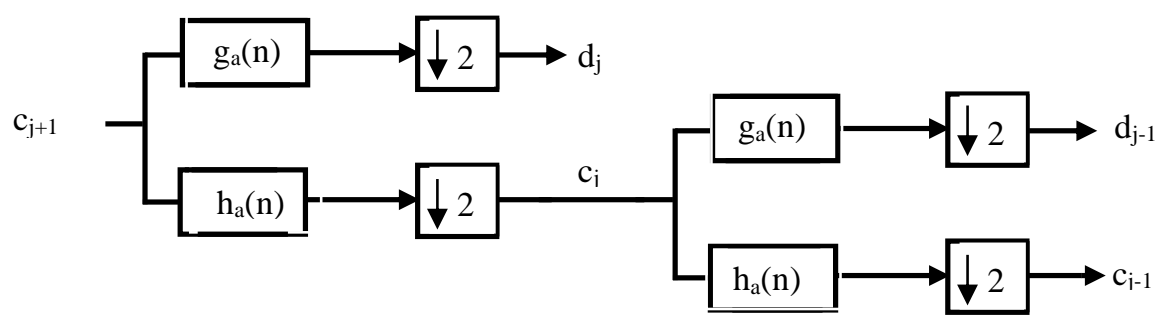

Fig. 7. Two levels DWT decomposition [1]

For the reconstruction process, the reversed methodology can simply be applied to the signal, so the detail and approximation components at the larger scale are fed back through the low and high pass filters respectively. Before that, the coefficients have to be upsampled by 2 , see Figure 8 [25, 27].

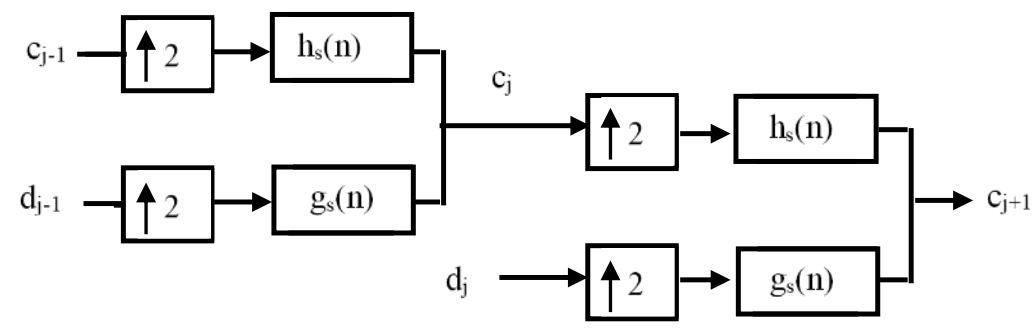

Fig. 8. Two levels DWT reconstruction [1]

\subsection{Correlation}

The similarity degree between two signals is utilized by correlation which has enormous applications such as sonar, radar, geology, etc. Let $x(n)$ and $y(n)$ be two different signals so cross-correlation $\left(\mathrm{r}_{\mathrm{xy}}\right)$ of these two signals can be calculated as:

$$
r_{x y}[m]=\sum_{-\infty}^{\infty} x[n] y[n-m]
$$

Where $m$ is the time shift (lag) parameter and the subscripts $x y$ of cross-correlation indicates the sequences being correlated. That's in Eq. 2, only the signal $y(n)$ is shifted from right to left with respect to $\mathrm{x}(\mathrm{n})$ signal when $m$ changes from positive to negative. When $\mathrm{x}(\mathrm{n})=\mathrm{y}(\mathrm{n})$ which is a special case, the operation is called autocorrelation. [28]:

\section{$3 \quad$ Results and Discussions}

\subsection{Dataset}

ECG signals are taken from MIT-BIH Arrhythmia Database version 3. It has 45 different records, 19 females and 26 males. This dataset contains 17 classes for normal 
sinus rhythm, AF ... etc. For each class, 10 -second sessions at least were collected. The recordings are digitalized at $360 \mathrm{~Hz}$ with a gain of $200 \mathrm{adu} / \mathrm{mV}$. The ECG signals were derived from one lead and all were formatted in mat format (Matlab)[29]. This work distinguishes between only two ECG classes: normal sinus rhythm and AF, where these two classes belong to 19 and 6 subjects, respectively. However, the data acquisition of one subject for the second class has a problem so it is discarded. Therefore, the data of only 5 subjects had been taken for two classes to balance the dataset. Table 1 illustrates the records used in each ECG class. After segmenting the ECG, 100 single heartbeats are extracted from each subject so the set of the heartbeats are 500 from 5 subjects of each class. 10-fold cross-validation was used in this work for evaluating the classification rates of the classifiers.

Table 1. ECG sessions of interest in each class.

\begin{tabular}{|l|l|}
\hline \multicolumn{1}{|c|}{ ECG class } & \multicolumn{1}{c|}{ Sessions number } \\
\hline Normal sinus rhythm & $100,101,103,105,106$ \\
\hline AF & $201,202,203,210,219$ \\
\hline
\end{tabular}

\subsection{Results}

The performance evaluation of the classifiers is based on the classifier prediction ratio for testing tuples correctly and incorrectly according to the following equation:

$$
A \text { ccuracy }=\frac{\text { number of correct predictions }}{\text { total number of predictions }}=\frac{\mathrm{TP}+\mathrm{TN}}{\mathrm{TP}+\mathrm{FP}+\mathrm{TN}+\mathrm{FN}}
$$

Where (TP) is the number of data tuples that are predicted correctly as a positive class while the false negative (FP) is the number of the data tuples which are predicted wrongly as a positive class. True negative (TN) represents the number of the data tuples that are predicted correctly as a negative class while the false negative (FN) depicts the number of the incorrect data tuples predicted as a negative class.

For the MLP classifier, the neurons of the hidden layer are experimentally specified. Some experiments were accomplished to determine the number of neurons that can produce the best performance (best classification accuracy with low SD). Each experiment was repeated 10 times for obtaining a more reliable result. Figure 9 illustrates the means of accuracies and SDs of the applied experiments. It is clear that the hidden layer of 22 nodes has attained the best performance. 


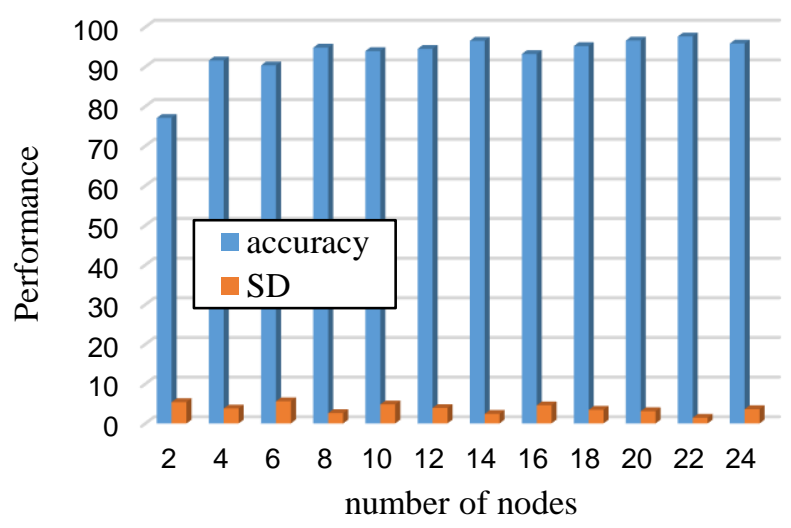

Fig. 9. MLP performances for a set of experiments to determine the number of nodes in the hidden layer

The classifications are repeated ten times for both classifiers in order to have more reliable results. Figure 10 highlights the classification rates for both the SVM and MLP classifiers. This figure shows that the MLP has recorded a larger classification rate of about $1.4 \%$ but it has more classification error around ten runs $(\mathrm{SD}=1.4)$. Therefore, SVM provides a more stable performance.

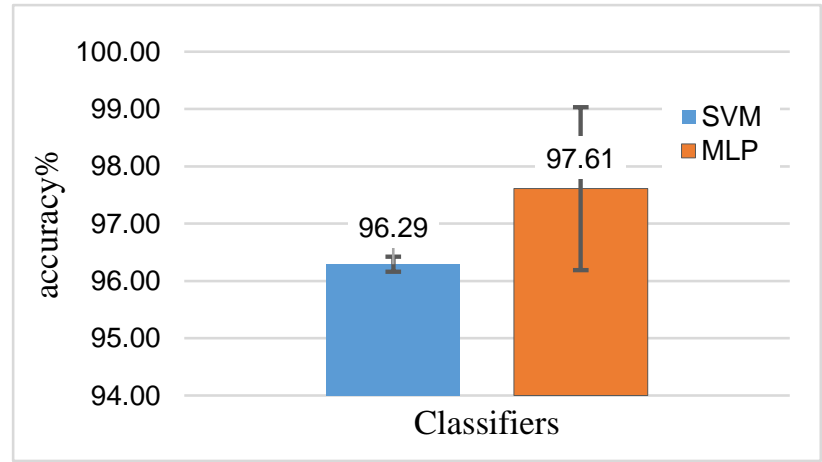

Fig. 10. Classification rate for SVM and MLP

The proposed ECG segmentation procedure has effect to the classification impact. This algorithm found the mean interval of ECG signal is 294 samples, 147 at both sides of the $\mathrm{R}$ point. This is 66 samples less than the expected interval (i.e., 360 samples). Figure 11 shows the difference between normal segmentation (single beat every one second) and the proposed ECG segmentation algorithm. 


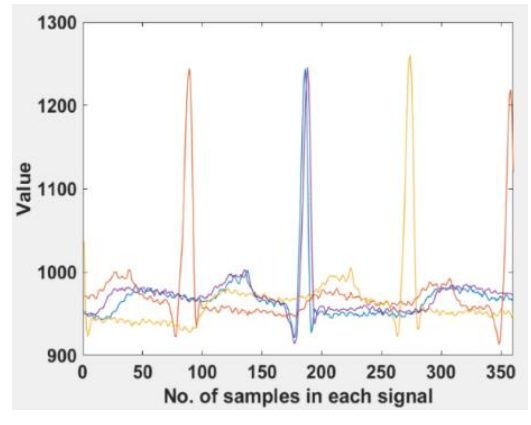

(a) normal segmentation

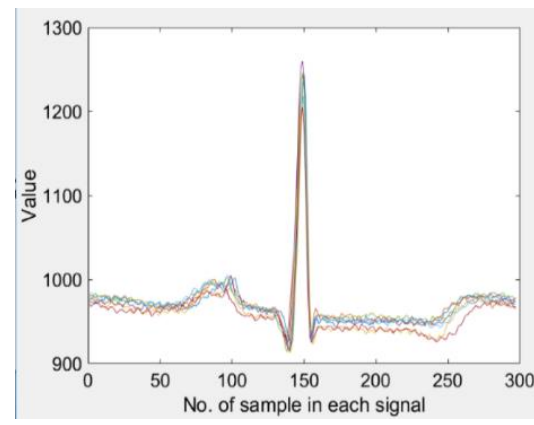

(b) the proposed segmentation algorithm

Fig. 11.ECG segmentation

The classification rates of two classifiers (SVM and MLP) were also examined and compared. Normal ECG segmentation procedure degrades the performances of our procedure to $77 \%$ and $80 \%$ respectively of the two classifiers. The low performance of the classifiers was caused by the synchronization of a single heartbeat (i.e. the set of heartbeats has different phases) see Figure 11 (a). The proposed method could improve the performance of the two classifiers to about $19 \%$ and $17 \%$ for the SVM and MLP, respectively. Figure 12 highlights the performance before and after applying the proposed segmentation algorithm.

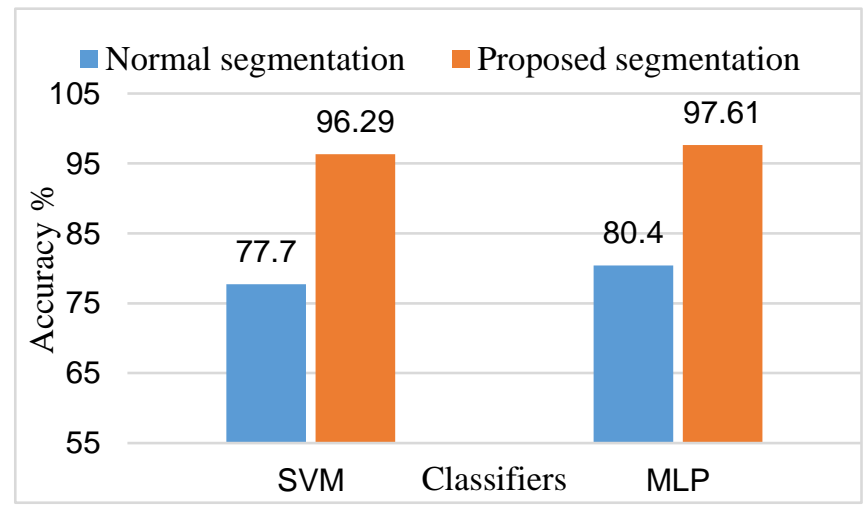

Fig. 12. The effects of the ECG segmentation procedure on the classification rates

In the proposed ECG segmentation algorithm, mean and SD are used to determine the threshold value but the ECG signal is corrupted with various artifact noises that influence the mean and the SD of the ECG signal, see Figure 13. Therefore, the algorithm uses the local mean and SD of the signal by windowing the signal with 360 samples $(1 \mathrm{sec}$.) window. Determining the $\mathrm{R}$ point is done by choosing the first single sample or the medial sample of the first successive samples that pass the threshold value. 
The reason for using the ECG signal as an exponent of 1.1 is to increase only the positive values of the signal exponentially, and this increases the opportunity that the Rpoint crosses the threshold value. Also, this prevents the negative values from disturbing the procedure, unlike other methods like the power of 2. A small base (1.1) was used to overcome the overflow problem of programming language variables. After determining the index of $\mathrm{R}$ points for the whole dataset and the mean interval of the single heartbeat, the algorithm can segment the ECG signal correctly and makes the R points lie at the center of the heartbeat.

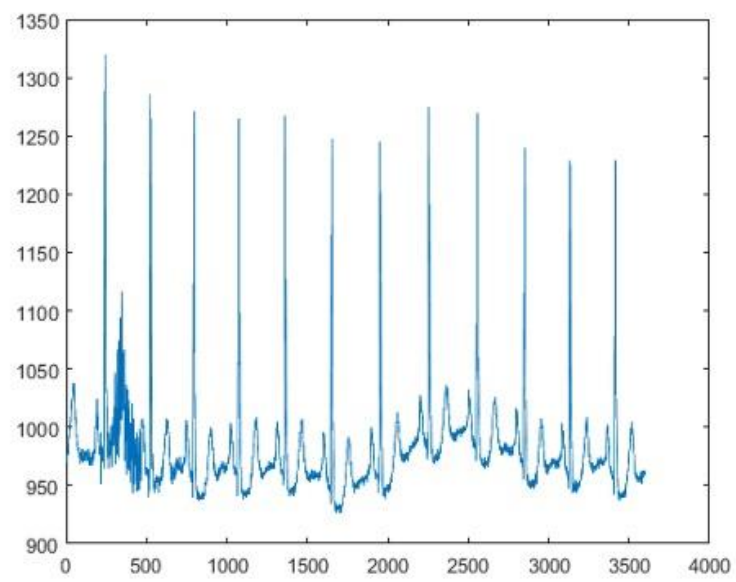

Fig. 13.ECG noisy signal

\section{Conclusion}

In real-time ECG systems, using the simplest methods to manipulate and extract features from the ECG signal for classification has crucial priority especially when ECG applications need quick response to rescue human life. Our preprocessing procedure is composed of using traditional analyzing methods such as cross-correlation, wavelet transform. Straightforward statistical methods (median, maximum ... etc.) were exploited to extract features from the preprocessed ECG signal. The extracted features are then tested using SVM and MLP. SVM provides a reliable classification rate and this is shown with a low classification error $(\mathrm{SD}=0.131)$, but its performance $(96.2 \%)$ is lower than MLP performance about (1.4\%). Segmenting the ECG signal has also effects on classification impacts for SVM and MLP. These effects are visible with the performance degradation of about $19 \%$ and $17 \%$ for SVM and MLP whenever excluding the proposed ECG segmentation algorithm. 


\section{$5 \quad$ References}

[1] C. Lastre-Dom-nguez, Y. S. Shmaliy and O. e. a. Ibarra-Manzano, "ECG Signal Denoising and Features Extraction Using Unbiased," Hindawi, BioMed Research International, 2019. https://doi.org/10.1155/2019/2608547

[2] M. Baydoun, L. Safatly, O. K. A. Hassan and e. al, "High Precision Digitization of PaperBased ECG Records: A Step Toward Machine Learning," IEEE, journal of translational engineering in health and medicine, vol. 7, November 2019. https://doi.org/10.1109/jtehm. 2019.2949784

[3] D. R. Seshadri, B. Bittel, D. Browsky and e. al, "Accuracy of the Apple Watch 4 to Measure Heart Rate in Patients with Atrial Fibrillation," IEEE, journal of translational engineering in health and medicine vol. 8, February 2020. https://doi.org/10.1109/jtehm.2019.2950397

[4] H. V. Huikuri, A. Castellanos and R. J. Myerburg, "Sudden death due to cardiac arrhythmias," New England Journal of Medicine, vol. 345, p. 1473-1482, 2001. https://doi.org/ $10.1056 /$ nejmra000650

[5] U. Erdenebayar, H. Kim, J.-U. Park, D. Kang and K.-J. Lee, "Automatic Prediction of Atrial Fibrillation Based on Convolutional Neural Network Using a Short-term Normal Electrocardiogram Signal," journal of korean medical science, 2019. https://doi.org/10.3346/ jkms.2019.34.e64

[6] A. Estrella-Gallego, M. Vazquez-Briseno, J. Nieto-Hipolito and e. al., "Monitoring Atrial Fibrillation Using PPG Signals and a Smartphone," iJOE, vol. 16, 2020. https://doi.org/ 10.3991/ijoe.v16i03.12871

[7] S. M. Anwar, M. Gul, M. Majid and M. Alnowami, "Arrhythmia Classification of ECG Signals Using Hybrid Features," Hindawi,Computational and Mathematical Methods in Medicine, 2018. https://doi.org/10.1155/2018/1380348

[8] N. A. Abdul-Kadir, N. M. Safri and M. A. Othman, "Atrial fibrillation classification and association between the natural," elsevier, International Journal of Cardiology, vol. 222, p. 504-508, 2016. https://doi.org/10.1016/j.ijcard.2016.07.196

[9] Roa'a M Al_airaji, Aljazaery Ibtisam A, Al_Dulaimi Suha Kamal, Alrikabi Haider TH Salim, and Informatics, "Generation of High Dynamic Range for Enhancing the Panorama Environment," Bulletin of Electrical Engineering, vol. 10, no. 1, 2020. https://doi.org/10. 11591/eei.v10i1.2362

[10] S. Celin and K. Vasanth, "ECG Signal Classification Using Various Machine Learning Techniques," Springer, Journal of Medical Systems, 2018. https://doi.org/10.1007/s10916018-1083-6

[11] Y. Ji, S. Zhang and W. Xiao, "Electrocardiogram Classification Based on Faster Regions with Convolutional Neural Network," MDPI, sensors, 2019. https://doi.org/10.3390/ $\underline{\mathrm{s} 19112558}$

[12] B. Guelta, R. Tlemsani, S. Chouraqui and M. Benouis, "An Improved Behavioral Biometric System based on Gait and ECG signals," International Journal of Intelligent Engineering and Systems, vol. 12, September, 2019. https://doi.org/10.22266/ijies2019.1231.14

[13] K. Naijit, "Emergency Notification Using Combination Algorithm with Recognition ECG Signal," iJOE , vol. 16, 2020. https://doi.org/10.3991/ijoe.v16i05.12707

[14] J. H. Al-Khalidy and R. R. Al-Nima, "Breast tumor classification using SVM," Tikrit Journal of Engineering Sciences, vol. 5, 2013.

[15] Hamid Mirvaziri and Zabihollah Saberi Mobarakeh, "Improvement of EEG-based motor imagery classification using ringtopology-based particle swarm optimization," Biomedical Signal Processing and Control,Elsevier, p. 69-75, 2016. https://doi.org/10.1016/j.bspc. $\underline{2016.10 .015}$ 
[16] S. Sanei and J. Chambers, EEG Signal Processing, England: John Wiley \& Sons Ltd, 2007.

[17] C. Seyidbayli, F. Salhi and E. Akdogan, "Comparison of Machine Learning Algorithms for EMG Signal Classification," Periodicals of Engineering and Natural Sciences, vol. 8, pp. 1165-1176, June 2020.

[18] Aljazaery Ibtisam A, Alrikabi Haider Th Salim, and Aziz Mustafa Rabea, "Combination of Hiding and Encryption for Data Security," International Journal of Interactive Mobile Technologies, vol. 14, no. 9, pp. 34-47, 2020. https://doi.org/10.3991/ijim.v14i09.14173

[19] M. L. Al -Dabag, N. Ozkurt and S. M. Al-Aimam, "Feature Selection and Classification of EEG Finger Movement Based on Genetic Algorithm," in 2018 Innovations in Intelligent Systems and Applications Conference (ASYU), Adana, 4-6 October 2018. https://doi.org/ 10.1109/asyu.2018.8554029

[20] S. Alseelawi, E. K. Adnan, H. T. Hazim, H. Alrikabi, and K. Nasser, "Design and Implementation of an E-learning Platform Using N-Tier Architecture," international journal of interactive mobile technologies, vol.14, issue.6, pp.171-185, 2020. https://doi.org/10.3991/ ijim.v14i06.14005

[21] T. Waili and M. G. e. a. Md Johar, "EEG Based Biometric Identification Using Correlation and MLPNN Models," iJOE, vol. 15, 2019.

[22] M. Li, W. Chen and T. Zhang, "Classification of epilepsy EEG signals using DWT-based envelope analysis and neural network ensemble," Biomedical Signal Processing and Control,Elsevier, pp. 357-365, 2017. https://doi.org/10.1016/j.bspc.2016.09.008

[23] P. S. Addison, The Illustrated Wavelet Transform Handbook Introductory Theory and Applications in Science, Engineering, Medicine and Finance, Edinburgh: Taylor \& Francis Group, 2017. https://doi.org/10.1201/9781315372556

[24] O. H. Yahya, H. Alrikabi, I. A. J. I. J. o. O. Aljazaery, and B. Engineering, "Reducing the Data Rate in Internet of Things Applications by Using Wireless Sensor Network," International Journal of Online and Biomedical Engineering (iJOE), vol. 16, no. 03, pp. 107-116, 2020. https://doi.org/10.3991/ijoe.v16i03.13021

[25] E. Aydemir, T. Tuncer and S. Dogan, "A Tunable-Q wavelet transform and quadruple symmetric pattern based EEG signal classification method," Medical Hypotheses, vol. 134, 2020. https://doi.org/10.1016/j.mehy.2019.109519

[26] J. G. Proakis, Digital Signal Processing, 4th Edition, New Jersey: Prentice Hall, 2006.

[27] Yahya Omar Hashim, ALRikabi Haider Th, Al_airaji Roa'a M, and Faezipour Miad, "Using Internet of Things Application for Disposing of Solid Waste," International Journal of Interactive Mobile Technologies, vol. 14, no. 13, pp. 4-18, 2020. https://doi.org/10.3991/ ijim.v14i13.13859

[28] M. L. Al- Dabag and N. Ozkurt, "EEG Motor Movement Classification Based On CrossCorrelation With Effective Channel," Signal, Image and Video Processing, Springer, 24 October 2018. https://doi.org/10.1007/s11760-018-1383-9

[29] C. S. Burrus, R. A. Gopinath and H. Guo, Introduction to Wavlets And Wavelet Transform, New Jersey: Printice Hall, 1998.

\section{Authors}

Mohand Lokman Aldabag completed his Ph.D. Program at Yasar University, Turkey. He received his B.E. in computer engineering degree from technical collegelMosul and his MSc. Degree in computer engineering from Mosul University, 1n 1998 and 2002 respectively. He worked at the department of technical computer engineering $\backslash$ Northern Technical university as an assistant lecturer in 2002-2012 and as a lecturer in 
the same university from 2012 till now. He published five articles in signal processing and hardware simulation of discrete wavelets. His research interests are biomedical signal processing, wavelet transform, and signal classification. (Northern Technical University, Technical Engineering College of Mosul, Mosul, Iraq). mohandaldabag@ntu.edu.iq

Haider Th. Salim ALRikabi He is presently Asst. Prof and one of the faculty College of Engineering, Electrical Engineering Department, Wasit University in Al Kut, Wasit, Iraq. He received his B.Sc. degree in Electrical Engineering in 2006 from the Al Mustansiriya University in Baghdad, Iraq. His M.Sc. degree in Electrical Engineering focusing on Communications Systems from California state university/Fullerton, USA in 2014. His current research interests include Communications systems with the mobile generation, Control systems, intelligent technologies, smart cities, and the Internet of Things (IoT). Al Kut city - Hay ALRabee, Wasit, Iraq. E-mail: hdhiyab@uowasit.edu.iq. The number of articles in national databases - 10, and the number of articles in international databases -20 .

Raid Rafi Omar Al-Nima received the BSc and MSc degrees in Technical Computer Engineering in 2000 and 2006, respectively. During 2006, he worked as an Assistant Lecturer in the Technical College of Mosul, Iraq. In 2011, he obtained the Lecturer's scientific title in the same college. In 2017, he accomplished his Ph.D. in the School of Electrical and Electronic Engineering at Newcastle University, UK. His research interests are in the fields of pattern recognition, security, artificial intelligence, and image processing. (Northern Technical University, Technical Engineering College of Mosul, Mosul, Iraq).

Article submitted 2020-10-12. Resubmitted 2020-12-12. Final acceptance 2020-12-16. Final version published as submitted by the authors. 